\title{
MODELLING RANDOM MEDIA
}

\section{DOMINIQUE JEULIN}

Centre de Morphologie Mathématique, Ecole des Mines de Paris, 35 rue Saint-Honoré, F77300 Fontainebleau, France e-mail: jeulin@cmm.ensmp.fr (Accepted July 17, 2002)

\begin{abstract}
Basic random structure models (random sets and random function models) are introduced for the simulation of images and of microstructures. Their implementation requires the use of image analysis tools defined in mathematical morphology. They can be used for solving problems of physics of random media.
\end{abstract}

Keywords: random structures, Boolean model, dead leaves, reaction diffusion, homogenization, fracture statistics, simulations, mathematical morphology.

\section{INTRODUCTION}

Many physical phenomena which occur in materials, geological and biological structures present heterogeneous properties at various scales, which can be accounted for by a probabilistic approach. Various types of models are available for describing and simulating heterogeneous structure. The principal types of models in the field of random images can be commonly classified as follows: i) stochastic point processes, to reproduce dispersions of small particles in a matrix; ii) random tessellations of space to generate granular structures, such as polycrystals; iii) random sets (binary), or multicomponent random sets, to represent porous media or mineralogical textures; iv) random functions (scalar or multivariate random functions), useful for grey level or color images, rough surfaces, and for chemical concentration mappings; v) random graphs to describe data on a network connecting vertices (roads, porous media, crack networks), and random trees for arborescent data (as seen in organs like lung, kidney (Fricout et al., 2002). After a recall of basic properties and characterization of random structures, we introduce some models of random sets and of random functions, and we shortly discuss their use to predict the physical behavior of random media.

\section{BASIC PROPERTIES OF RANDOM MEDIA}

\section{Morphological criteria}

The main morphological types of data to quantitatively describe random structures can be classified according to the main following morphological criteria (Matheron, 1967; Serra, 1982; Stoyan et al., 1987; Jeulin, 1998b; 2000a; 2001):
- Basic measures (volume fraction, surface area, integral of mean curvature, ... ) are global quantitative measurements with suitable stereological properties (they can be estimated for three-dimensional objects from lower dimensional sections (1D or 2D such as images).

- Sizing of objects in a microstructure can be obtained as a size distribution (with some restrictions on the shape, such as a limitation to spheres, concerning estimation from 1D or $2 \mathrm{D}$ to 3D); sizing of media of any shape (including connected networks) can be performed by the opening (erosion followed by a dilation) or closing (dilation followed by an erosion) morphological operations by convex structuring elements (Matheron, 1975; Serra, 1982).

- Distribution in space is very important to account for the presence of scales:

- Clustering of objects may be studied by the probability distribution of distances $r$ from a random point $x$ outside of the objetcs to their boundary, $F_{x}(r)$ (estimated from volume fraction measurements after dilations with increasing sizes)

- Scales and their superposition can be quantified from second order statistics, based on the covariance, from which nested structures such as clusters, clusters of clusters, repulsion effects, as well as periodicity in images are easily detected; the integral range is a standard measure of the size of a representative volume element of the microstructure described by a stationary and ergodic random structure: in the space, a volume $V$ is made of $n=V / A_{3}$ volume elements, inside which the average 
values of a $R F Z(x)$ are uncorrelated random variables, and therefore the integral range $A_{3}$ is a good measure of the notion of scale. It is a convenient measurement of the size of a RVE (representative volume element) of a stationary and ergodic random structure.

- Anisotropy is assessed from directional measurements, such as the variation of the covariance or of the chord size distribution with their orientation.

- Connectivity has a major incidence on the physical properties of composites made of phases with a high contrast, like microcracks or voids.

The principle of morphological measurements in two steps was proposed by Serra (1982):

- a morphological transformation $\Phi$ is applied to the structure (using image analysis);

- some measurement is performed on the transformed object.

The choice of measures and of transformations is made according to morphological criteria, as detailed in references.

\section{Characterization of random structures}

When deciding to use a probabilistic approach, it is important to be able to characterize a random medium by appropriate tools. A generalization of the notion of random variable was developed by G. Matheron for random closed sets RACS, through the Choquet capacity $T(K)$, defined on the compact sets $K$ (Matheron, 1969; 1975). If $A$ is a random closed set, then

$$
\begin{aligned}
T(K)=P\{K\ulcorner A \neq \emptyset\}=1-P\{ & \left.K \subset A^{c}\right\} \\
& =1-Q(K) .
\end{aligned}
$$

In practice, geometrical figures (finite sets of points, segments, discs, spheres ... ) are used for the compact set $K$, in order to test various morphological properties of a heterogeneous structure. When operating in the euclidean space, the Choquet capacity is connected to the basic operations of mathematical morphology, namely the dilation and the erosion (related to the Minkowski addition $\oplus$ and substraction $€$ ), and we have for a compact set $K$ translated in $x$, where $\check{K}=$ $\{-x, x \in K\}$ :

$$
\begin{aligned}
& T\left(K_{x}\right)=P\left\{K_{x} \cap A \neq \emptyset\right\}=P\{x \in A \oplus \check{K}\}, \\
& Q\left(K_{x}\right)=P\left\{K_{x} \subset A^{c}\right\}=P\left\{x \in A^{c} \in \check{K}\right\} .
\end{aligned}
$$

When $K=\left\{x_{1}, x_{2}, \ldots, x_{n}\right\}$, we obtain the spatial law of $A$ :

$$
T(K)=1-P\left\{x_{1} \in A^{c}, x_{2} \in A^{c}, \ldots, x_{n} \in A^{c}\right\}
$$

The spatial law cannot completely characterize the RACS $A$, since in that case we have $T(K)=0$ for any stationary point process, and therefore we have to use for instance as compact set $K$ the closed ball with radius $r, B(r)$, involving an undenumberable set of points. However, the spatial law can be used to estimate bounds of the effective physical properties of random media (Jeulin, 1998b; 2001). Random closed sets have been extended to upper semi continuous random functions (usc RF) (Matheron, 1969; Norberg, 1986; Verwaat, 1988). For a usc RF $Z(x)$, a generalization of the Choquet capacity, $T(g)$, can be defined (Jeulin, 1991) on the lower semi continuous functions (lsc) $g$ with compact support $K$ :

$$
T(g)=P\left\{x \in D_{Z}(g)\right\} ;
$$

where

$$
D_{Z}(g)^{c}=\{x ; Z(y)<g(y-x), \forall y \in K\} .
$$

$D_{Z}(g)^{c}$ is the set of points $x$ where the function $Z(y)$ remains below the test function $g$, when its support $K$ is translated in $x$. A particular and common case is the spatial law defined for a finite number of points:

$$
\begin{aligned}
& F\left(x_{1}, x_{2}, \ldots, x_{n}, z_{1}, z_{2}, \ldots, z_{n}\right)= \\
& \quad P\left\{Z\left(x_{1}\right)<z_{1}, Z\left(x_{2}\right)<z_{2}, \ldots, Z\left(x_{n}\right)<z_{n}\right\} .
\end{aligned}
$$

The spatial law is deduced from Eq. 2 as $1-T(g)$ when $g\left(x_{i}\right)=z_{i}$ and elsewhere $g(x)=+\infty$. Other cases include the random functions

$$
Z_{\vee}(K)=\vee_{x \in K}\{Z(x)\} ; Z_{\wedge}(K)=\wedge_{x \in K}\{Z(x)\}
$$

which are generated by changing the support via the supremum $(\vee)$ and infimum $(\wedge)$. This is used in applications to Fracture Statistics Models based on the weakest link assumption, and to the statistics of extremes. We have to stress the fact that the functional $T(K)$, (or $T(g)$ ), connects theory and experiments; it is used to estimate the parameters of a model and to test its validity. The functional $T$ is obtained by theoretical calculation, or by estimation on simulations of the random structure, or on samples of the real structures. The estimation of the functional $T(K)$ is easily performed from the implementation of the basic operations of mathematical morphology, namely erosions or dilations. The morphological content of the Choquet capacity, depending on the choice of the compact set $K$, is detailed in (Serra, 1982; Jeulin, 1991; 2000; 2001). 


\section{RANDOM SETS MODELS}

\section{Point processes}

Point processes are probably the simplest kind of random structure that we could imagine (Stoyan et al., 1987). They can be the first step in the construction of more general models, that are called grain models. Some of these models are presented in (Jeulin, 1997). The most popular random point process model is the Poisson process. The non homogeneous Poisson point process in $R^{n}$ with a regionalized intensity $\theta(x)\left(x \in R^{n} ; \theta \geq 0\right)$ is such that the numbers $N\left(K_{i}\right)$ are independent random variables for any family of disjoint compact sets $K_{i}$.

$N(K)$ is a Poisson random variable with parameter $\theta(K)$

$$
\begin{gathered}
\theta(K)=\int_{K} \theta(d x) \\
P_{n}(K)=P\{N(K)=n\}=\frac{\theta(K)^{n}}{n !} \exp (-\theta(K))
\end{gathered}
$$

Starting from a point process, more general models, called grain models, can be generated:

- The Boolean model

- The dead leaves model

- Random function models

\section{The Boolean model}

The Boolean model (Matheron, 1967; 1975; Serra, 1982; Stoyan et al., 1987) in the Euclidean space $R^{n}$ with the Lebesgue measure $\mu_{n}$ (i.e; the volume in $R^{3}$ or the area in $R^{2}$ ), is obtained by implantation of random compact primary grains $A^{\prime}$ (with possible overlaps) on Poisson points $\mathrm{x}_{k}$ with the intensity $\theta$, giving the random set: $A=\cup_{x_{k}} A_{x_{k}}^{\prime}$ (this construction is illustrated in Fig. 1, where a material microstructure (left) is compared to a realization of a section of a Boolean model of spheres (right)). By construction, the morphologies of the two phases $A$ and $A^{c}$ of this medium are asymmetric: $A$ is representing a collection of random aggregates, embedded in a matrix $A^{c}$. For this model, we have in the stationary case, with $q=$ $P\left\{x \in A^{c}\right\}$ :

$$
\begin{array}{r}
T(K)=1-Q(K)=1-\exp \left(-\theta \bar{\mu}_{n}\left(A^{\prime} \oplus \check{K}\right)\right) \\
=1-q^{\frac{\bar{\mu}_{n}\left(A^{\prime} \oplus \check{K}\right)}{\bar{n}_{n}\left(A^{\prime}\right)}}
\end{array}
$$

where $q=\exp \left(-\theta \bar{\mu}_{n}\left(A^{\prime}\right)\right)$ and $\bar{\mu}_{n}$ is the expected value of $\mu_{n}$ over the realizations of the primary grain
$A^{\prime}$. This expression results from the fact that the number of primary grains hit by $K$ follows a Poisson distribution with mean $\theta \bar{\mu}_{n}\left(A^{\prime} \oplus \check{K}\right)$. A particular case of Eq. 7 gives the covariance $Q(h)$

$$
\begin{aligned}
Q(h)=P\left\{x \in A^{c}, x+h \in A^{c}\right\} & \\
= & q^{2} \exp (\theta K(h))=q^{2-r(h)}
\end{aligned}
$$

with the geometrical covariogram

$$
K(h)=\bar{\mu}_{n}\left(A^{\prime}\left\ulcorner A_{-h}^{\prime}\right) \text { and } r(h)=K(h) / K(0) .\right.
$$

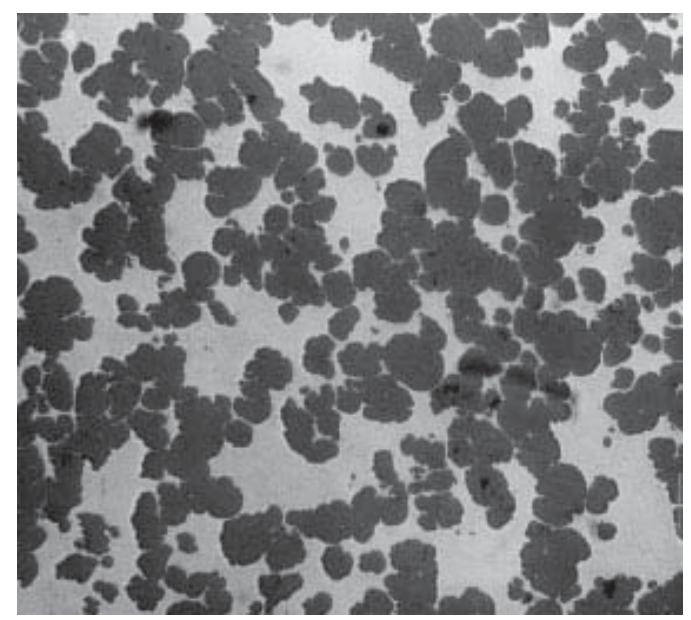

$\mathrm{Fe}$ (black) Ag (grey) alloy

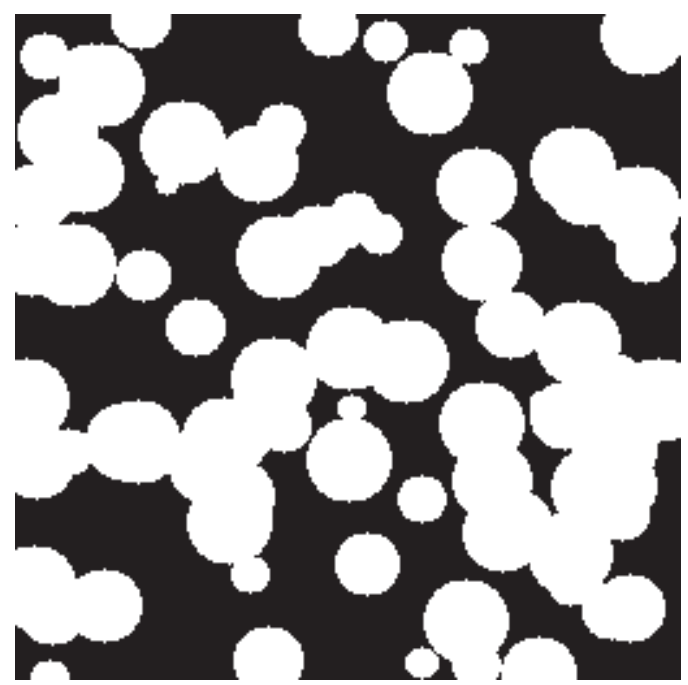

Boolean spheres $V_{u}=0.5$

Fig. 1. Example of material simulated by a Boolean model of spheres (Bretheau and Jeulin, 1989).

The Boolean model is a good candidate to simulate interconnected media since overlaps between grains are obtained with an increasing intensity $\theta$, as the result of the locations of the Poisson 
points. For instance, it mimicks quite well a sintered microstructure (compare a real and a simulated morphology in Fig. 1). The model is identified in practice from Eq. 7 connecting the average properties of the images of the texture $A$ to the average properties of the primary grain $A^{\prime}$. It enables us to estimate the parameters of the model, namely the intensity $\theta$, and the probabilistic properties of the primary grain $A^{\prime}$ (such as the distribution function of its diameter for spheres) from the experimental measurement of $T(K)$.

When the grain and the set $K$ are convex, the expression $\bar{\mu}_{n}\left(A^{\prime} \oplus \check{K}\right)$ is a polynomial of degree $k$, for $K$ with dimension $k$ (it is easy to check this point from experimental data for $T(K)$ ), with coefficients proportional to average properties of $A^{\prime}$ (volume, surface area, integral of mean curvature). It is in general not possible to derive the full probabilistic properties of the primary grains from the information obtained by convex sets $K$. For instance, using a segment $l$, we have $\left(K^{\prime}(h)\right.$ being the derivative of the covariogram with respect to $h$ )

$$
\begin{aligned}
Q(l) & =\exp \left(-\theta \bar{\mu}_{n}\left(A^{\prime} \oplus l\right)\right) \\
& =\exp \left(-\theta\left(K(0)-l K^{\prime}(0)\right)=q^{1-l r^{\prime}(0)},\right.
\end{aligned}
$$

which is commonly used to test the validity of the assumption "Boolean model with convex grains". One must anyway be careful with this test, since other models, such as the Poisson mosaic, produce exponential functions for $Q(l)$ (Aubert and Jeulin, 2000).

Using non connected sets (such as two or three points), more complete information on the random grain $A^{\prime}$ is obtained, since in that case $\bar{\mu}_{n}\left(A^{\prime} \in \check{K}\right)$ is derived from Eq. 7. Using two points, we can estimate the average geometrical covariogram of the primary grain through Eq. 8. For instance, the distribution function $f(a)$ of the diameters of random spheres is theoretically available from Eqs. 8 and 9. In the general case, it involves the numerical calculation of second order derivatives, which is an unstable procedure. Therefore it is wise to use prior parametric distribution functions $f(a)$ and to estimate their parameters from $r(h)$ using a procedure such as least squares, as was done for spheres (Bretheau and Jeulin, 1989) and for ellipsoids (Charollais et al., 1997).

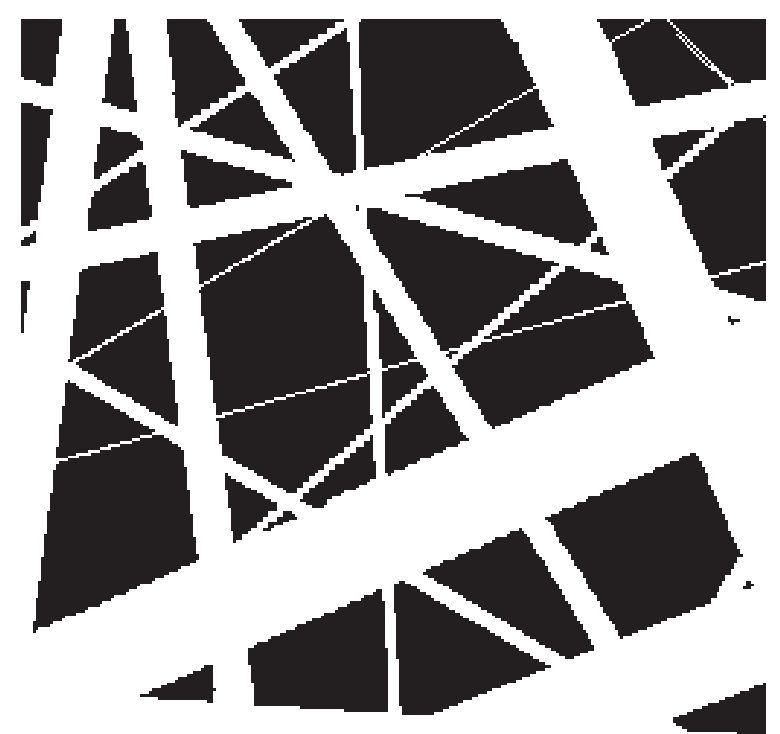

Fig. 2. Simulation of Poisson fibers or strata.

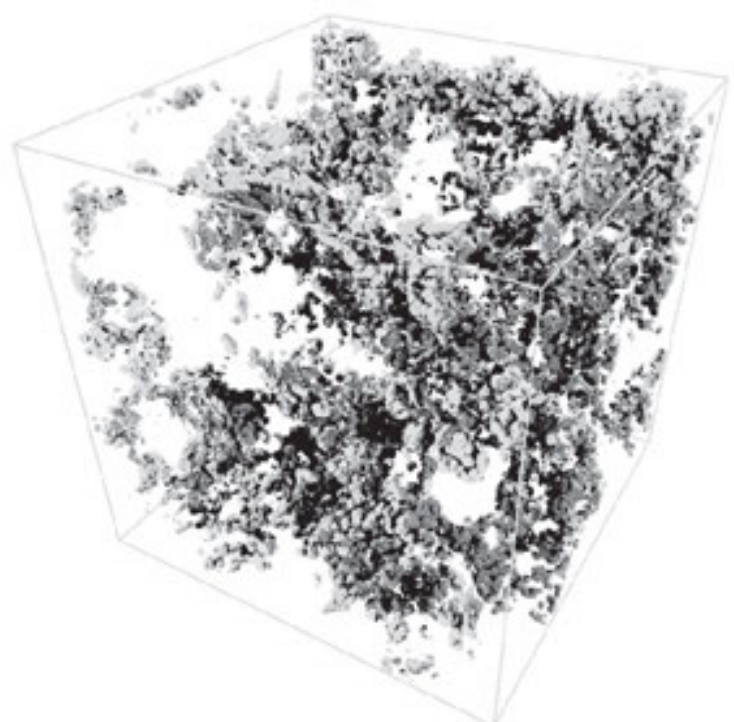

Fig. 3. Simulation of a carbon black nanocomposite by intersection of 3 scales Boolean models of spheres (identification from thick sections) (Savary et al., 1999).

Other examples of Boolean models are of interest: when $A^{\prime}$ is a point process with a compact support, the resulting Boolean model is the Neyman-Scott point process, which reproduces clusters; replacing the Poisson points by Poisson varieties (Matheron, 1975), i.e. affine subspaces such as lines or planes in $R^{3}$ which can be considered as Poisson point processes in appropriate parametric spaces, enables us to generate random set models with fiber or strata textures (Jeulin, 1991; 2001), which percolate for any volume fraction (Fig. 2). Note that these simulations 
can also be considered as 2D projections of Poisson cylinder fibers in the 3D space, as used for the morphology of wood composites (Delisée et al., 2001). In (Savary et al., 1999), combinations of Boolean models of spheres involving multiple scales, were used for the simulation of carbon black composites; the identification of the parameters of the model was made from the covariance of thick sections of specimens from electron microscope images (Fig. 3).

\section{The color dead leaves model}

The dead leaves model (Jeulin, 1987; 1989; 1991; 1997) is obtained sequentially by implantation of random primary grains $A_{i}^{\prime}(t)$ with different colors (two in the binary case) on a Poisson point process. Two constructions have been proposed: to every point $x$ is assigned a color which is (i) the first or (ii) the last color occurring during the sequence. In this way, asymmetric random sets are eventually obtained if two different families of primary grains are used for $A$ and for $A^{c}$ (in the case of a primary grain $A_{i}^{\prime}$ independent of the time $t$, and implanted with a constant intensity $\theta_{i}$, the space $R^{n}$ becomes totally covered as $t \rightarrow+\infty$ ). The shape of the resulting cell is non convex, due to the overlaps occurring during the construction of the model.

Random primary grains of various colors, $A_{i}^{\prime}(t)$, produce multi component random sets, useful to simulate random media with crystals of different phases. They are symmetric when the same grains are used for every color, as for the case of Fig. 4a, b. Probabilistic properties (mainly covariances and three points moments) are expressed as a function of the time of observation $t$ of the process (Jeulin, 1987; 1989; 1991; 1997). Considering the "intact" grains (uncovered by other grains when the sequence is stopped), a model of non overlapping particles is generated. The size distribution of the non overlapping particles, as well as their volume fraction can be estimated (Jeulin, 1993; 1998b).

In the case of a single type of convex symmetrical grain (for instance a sphere with a constant radius, or a cube with a fixed orientation), the resulting area fraction in $R^{2}$ and volume fraction in $R^{3}$ are given for the homogeneous model by 0.25 and 0.125 , respectively. For discs in $R^{2}$ (as in Fig. 5) or for spheres in $R^{3}$, the centres generate a standard hard core point process (Stoyan et al., 1987). Their covariance presents typical oscillations, which can never be the case for a Boolean model. Their theoretical expression is given in (Gille, 2002) for spheres.

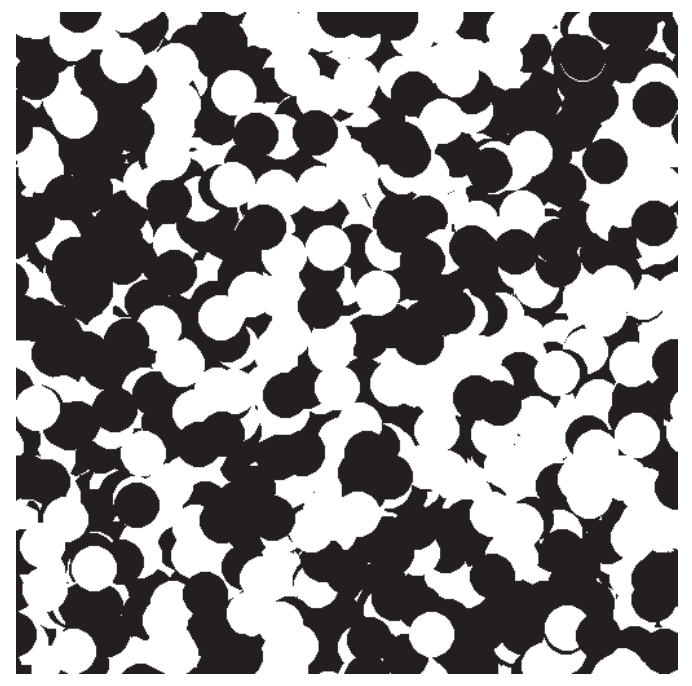

(a)

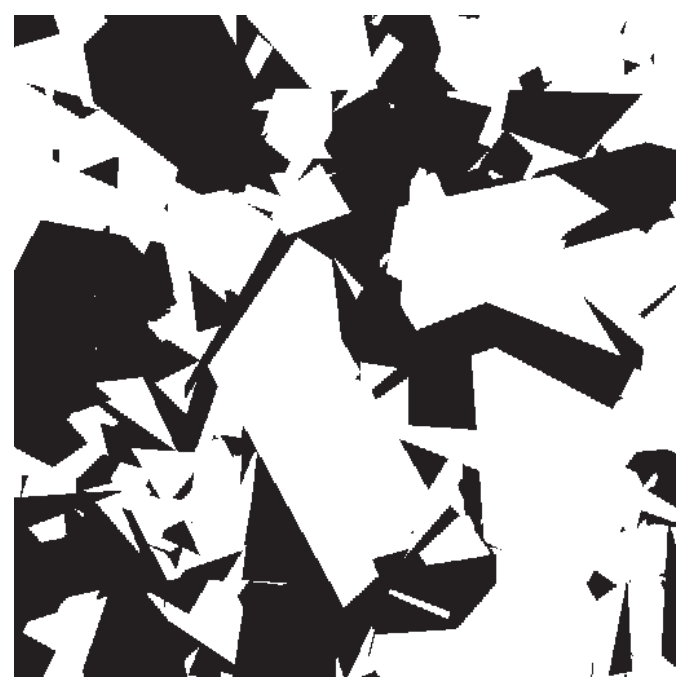

(b)

Fig. 4. Dead leaves models of discs $(a)\left(P_{i}=0.5\right)$ and of Poisson polyhedra $(b)\left(P_{i}=0.5\right)$.

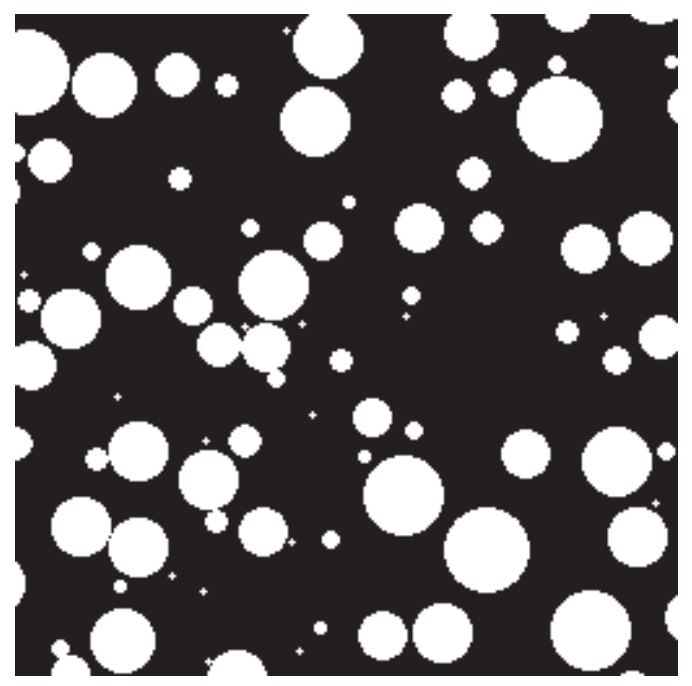

Fig. 5. Non overlapping discs built from the dead leaves model. 


\section{RANDOM FUNCTION MODELS}

The previous random set models have their continuous versions, as random functions (RF): the Boolean RF (Jeulin and Jeulin, 1981; Serra, 1988; Jeulin, 1991) considers the random implantation of primary random functions on points of a Poisson point process. In that case the $\cup$ operation for overlapping grains is replaced by the supremum $(V)$ or by the infimum $(\wedge)$. This type of model is very useful for applications to fracture statistics (Jeulin, 1991; 1994; 1998b; 2001), and to simulate rough surfaces. Examples of simulations are given in Fig. 6a, b.

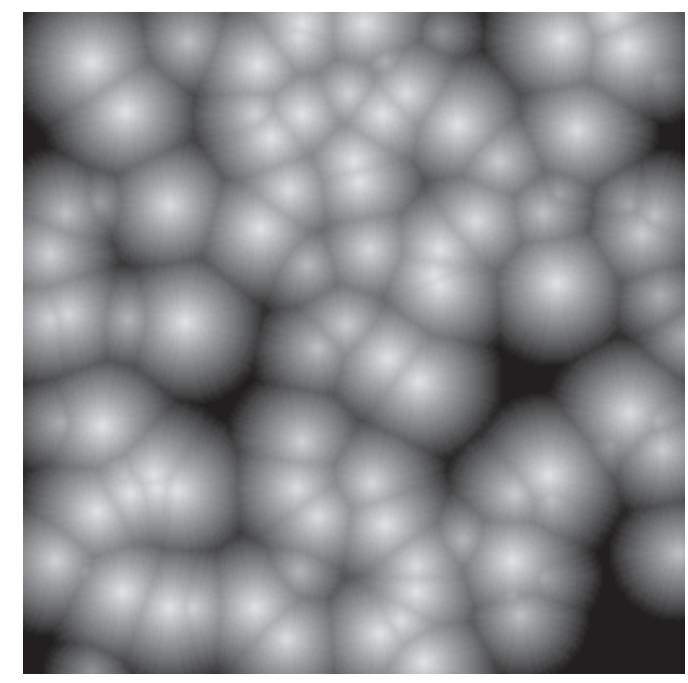

(a)

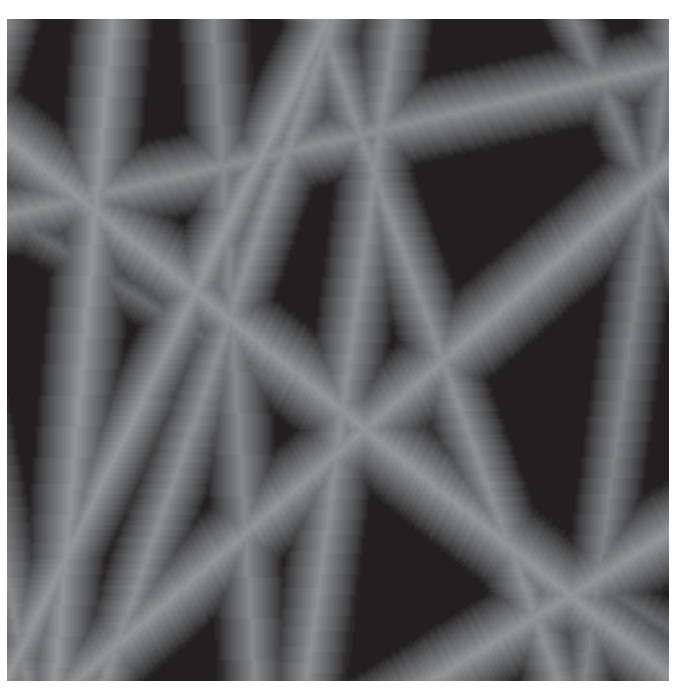

(b)

Fig. 6. Boolean RF with cone primary grains (a) and Boolean Variety RF (b).

Starting from a sequence of primary RF's $Z_{t}^{\prime}$, implanted according to the supremum with the intensity $\theta(t)$ we have

$$
\begin{aligned}
& 1-T(g)=Q(g)= \\
& \quad \exp \left(-\int_{R} \bar{\mu}_{n}\left(D_{Z_{t}^{\prime}}(g)\right) \theta(d t)\right) .
\end{aligned}
$$

As for the Boolean random set, the properties of the RF $Z$ are deduced from average properties of the primary function $Z_{t}^{\prime}$, through the set $D_{Z_{t}^{\prime}}(g)$ and the intensity $\theta(d t)$. Again, the model can be identified by means of the experimental $T(\mathrm{~g})$ and of its theoretical expression given in Eq. 11. As particular cases, are obtained the spatial law and the change of support by the supremum. If $g\left(x_{i}\right)=z_{i}$ in points $x_{i}(i=1,2, \ldots, n)$, else $g(x)=+\infty$, we get the spatial law:

$$
\begin{aligned}
& 1-T(g)=P\left\{Z\left(x_{1}\right)<z_{1}, \ldots, Z\left(x_{n}\right)<z_{n}\right\} \\
= & \exp \left(-\int_{R} \bar{\mu}_{n}\left(A_{Z_{t}^{\prime}}\left(z_{1}\right)_{x_{1}} \cup \ldots \cup A_{Z_{t}^{\prime}}\left(z_{n}\right)_{x_{n}}\right) \theta(d t)\right) .
\end{aligned}
$$

If $Z_{\vee}(K)=\vee_{x \in K}\{Z(x)\}$ and if $A_{Z_{t}^{\prime}}(z)=\left\{x, Z_{t}^{\prime}(x) \geq z\right\}$ we have

$$
P\left\{Z_{\vee}(K)<z\right\}=\exp \left(-\int_{R} \bar{\mu}_{n}\left(A_{Z_{t}^{\prime}}(z) \oplus \check{K}\right) \theta(d t)\right) .
$$

Similarly, the dead leaves random sets models have their counterpart as random functions. The dead leaves $\mathrm{RF}$ is used to reproduce scanning electron micrographs, and provides algorithms to estimate the morphological properties of powders (Jeulin, 1993) (Fig. 7). Explicit expressions are available for bivariate distributions and for the change of support by infimum (Jeulin, 1989).

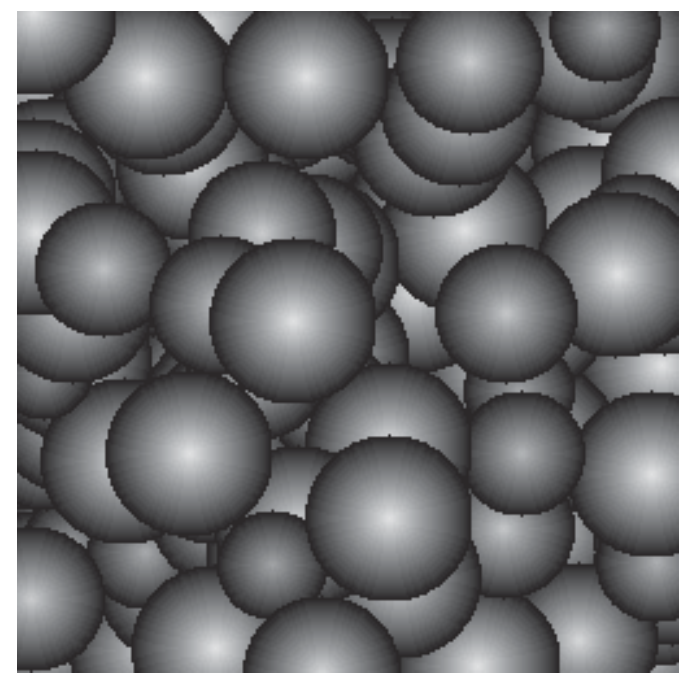

Fig. 7. Dead leaves $R F$ with cone primary grains. 


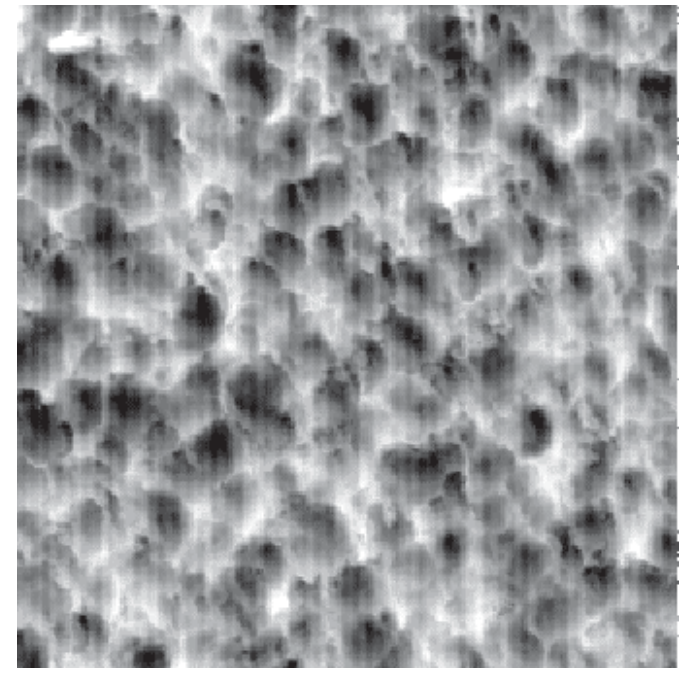

(a)

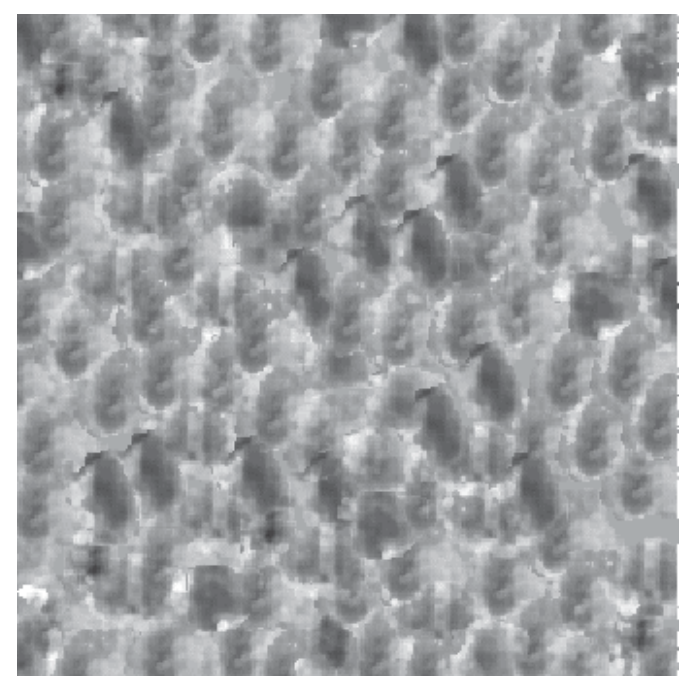

(b)

Fig. 8. Rough surface obtained by electroerosion (EDT texture) (a), and its simulation (b). (IRSID) $1.28 \times 1.28$ $\mathrm{mm}^{2}$.

These models and their parents such as Sequential Alternate RF (reproducing depositions and abrasions) are commonly used for the simulation of random surfaces (Jeulin, 1998a). Such models are useful for plate users (in steel and in car industry) and for mechanical parts users (to study the tribological or friction behavior of rough surfaces (Decencière and Jeulin, 2001)). In Fig. 8 is presented a rough surface obtained by electroerosion, and its simulation (Jeulin and Laurenge, 1996a). In a companion study (Jeulin and Laurenge, 1996b), the transfer of roughness to the plate during the skin-pass process was predicted by simulation of the indentation. Multivariate versions of models of random functions are used for color images, vector or tensor random fields (Jeulin, 1991). Concerning simulations, interesting developments are given in (Lantuéjoul, 2002), where in particular is detailed the generation of realizations of models satisfying experimental constraints, such as for instance given values at some data points.

Finally, Reaction-Diffusion random functions models are obtained as solutions of stochastic non linear parabolic partial differential equations. They model the competition between chemical interaction between species and their transport by diffusion. In the presence of $N$ chemical species $i$, the concentrations $Z_{i}(x, t)$ are solutions of a set of non linear partial differential equations, $D_{i}$ being the coefficient of diffusion of species $i$ :

$$
\frac{\partial Z_{i}(x, t)}{\partial t}=D_{i} \Delta Z_{i}(x, t)+F_{i}\left(Z_{1}, Z_{2}, \ldots, Z_{N}\right) .
$$

In (Decker and Jeulin, 1999; Decker, 1999), they are used to simulate spatial-time random structures, starting from a white noise as initial conditions, and using periodic boundary conditions. They produce sequences of images, generating complex morphologies which are well suited to natural textures. As opposite to previous models, which were based on the Poisson point process and on the use of primary grains, almost no results are available concerning their probabilistic properties, which is a major drawback for their practical use in applications. However, when sequences of images are available, the partial derivatives with time $t$ and with space coordinates $x$ can be estimated directly on images. In that case, the coefficients of diffusions $D_{i}$ and the coefficients of polynomial functions $F_{i}$ can be estimated by regression from the data available on pixels or voxels, using Eq. 14. An example of simulation is given for the complex time-dependent Ginzburg-Landau equation. It is a two species model $\left(Z_{1}\right.$ and $\left.Z_{2}\right)$ driven by the following equation:

$$
\frac{\partial Z}{\partial t}=D \triangle Z+A Z-B|Z|^{2} Z,
$$

with

$$
\begin{aligned}
& Z=Z_{1}+i Z_{2} \\
& A=\alpha+i \gamma \\
& B=\beta+i \delta .
\end{aligned}
$$

This equation admits spiral wave solutions, with oscillating and rotating behaviors. From 3D simulations, one can clearly notice the presence of a population of spiral structures, as illustrated in Fig. 9a, b, c. Their interaction can be displayed by animations reproducing the evolution with time of the solution of Eq. 15 (compare images at two times $t$ in Fig. 9b, c). 


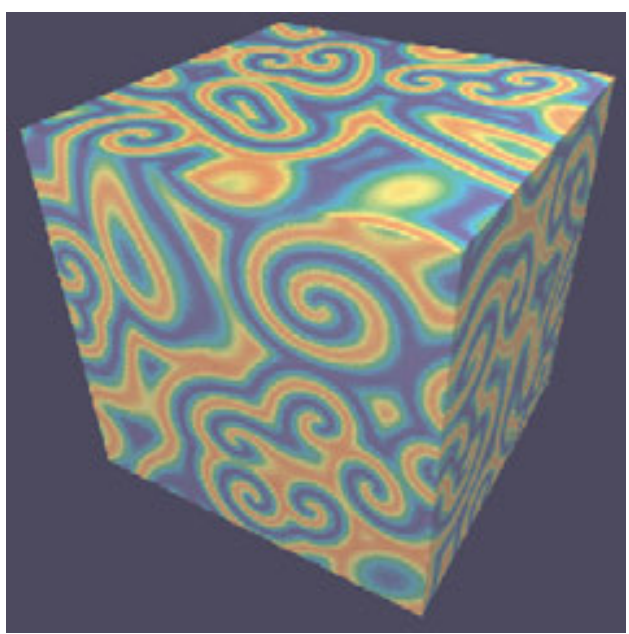

(a)

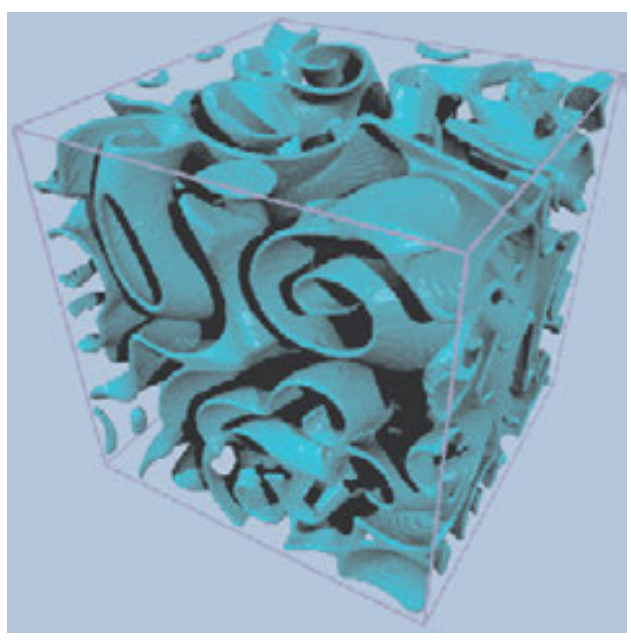

(b)

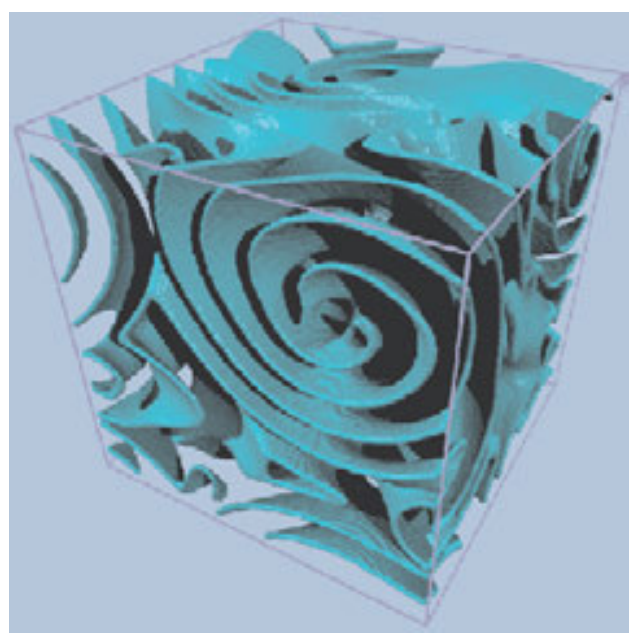

(c)

Fig. 9. Reaction Diffusion model built from the simulation of Ginzburg-Landau equations; component $Z_{1}$ (a); binary images obtained by thresholding $\left(Z_{1}(x, t)>0.9\right)$ after $2000(b)$ and $10000(c)$ iterations (Decker and Jeulin, 1999; Decker, 1999).

\section{PHYSICS OF RANDOM MEDIA}

Models of random media can be efficiently applied to the prediction of the macroscopic behaviour of a physical system from its microscopic behaviour. Firstly, they are used to estimate the effective properties (namely the overall properties of an equivalent homogeneous medium, which is the solution of a homogenization problem) of random heterogeneous media from their microstructure (Matheron, 1967). A first approach, using variational principles, provides bounds of the effective properties for linear constitutive equations. For instance, third order bounds (from third order central correlation functions) are derived for some random sets models (Jeulin and Le Coënt, 1996; Jeulin and Savary, 1997; Jeulin, 1998b; 2001). This was used to predict the effective elastic modulus of plaster, using a morphological description by a Boolean model (Jeulin et al., 2001). We could also show that a multiscale superposition of Boolean strata, as illustrated by Fig. 2 for one scale, generates two-phase random media with optimal effective properties (Jeulin 2001).

A second approach to estimate the effective behaviour is based on numerical simulations techniques like finite elements (FE) applied to realizations of random media (Decker and Jeulin, 2000). In (Barbe et al., 2001), this is worked out for the plastic behaviour of polycrystals from 3D simulations of random Voronoi tessellations. The main advantage of this technique is to provide to the computer 3D microstructures depending from controlled parameters, which would be difficult to obtain from experimental data. Similarly, transport properties (permeability, coefficient of macroscopic diffusion) of heterogeneous media (e.g. porous media) can be estimated by random walks or by geodesic propagations on 3D specimens (Decker et al., 1998). A promising technique concerns the use of numerical solutions of the field in random media by Fast Fourier Transforms, as originally proposed for the elastic properties of composites (Moulinec and Suquet, 1994), and more recently developed for the estimation of the dielectric properties of random structures (Eyre and Milton, 1999; Delarue, 2001). When using Monte carlo simulations, the statistical representativity of the results must be ensured. This point is illustrated in a study of fluctuations of effective properties in finite domains, as a function of the scale and of the contratst of thermal and of elastic properties of twophase random media based on a Voronoi mosaic (Kanit et al., submitted).

Another class of problems of interest concerns fracture statistics models (Jeulin, 1991; 1994; 1998b; 2001). The main purpose of this approach is to 
estimate the probability of fracture of a specimen under a given solicitation. Differently from the case of effective properties, there is in fracture a great sensitivity to local defects (with a large effect of tails of the distributions on the macroscopic fracture behaviour). One of the main points of fracture statistics approaches deals with size effects. For this, it is necessary to use models reproducing, at a point scale, the variations of a fracture criterion. For various fracture criteria, the probability of fracture is worked out analytically, as a function of the loading conditions and of the parameters of the random structure models. They can be tested at different scales (including the microscopic scale, by use of image analysis). The diversity of the obtained theoretical distributions for fracture statistics offers new possibilities for the microstructure based interpretation and modelling of mechanical data obtained on materials. For damaging materials at different scales, like fiber composites or metals under a ductile fracture, models of random damage were proposed, based on the statistics of point defects, the Boolean model, and an assumption of uniform load sharing during the progression of damage (Jeulin, 2000b). For damaging materials, the fracture process can also be studied by means of numerical simulations, combining FE calculations and Monte Carlo simulations of microstructures.

\section{CONCLUSION}

Random models of structures may be of interest to simulate complex images, such as the microstructures of materials or of biological specimens. The proposed approach, based on measurements obtained by image analysis and using basic transformations of mathematical morphology such as dilations or erosions, makes it possible to test and select appropriate models, to estimate their parameters, and to produce simulations. Beyond this descriptive power, they are used for the prediction of the overall physical behaviour or of the fracture statistics of random media, such as encountered in materials science. Another use of these models concerns the synthesis of textures for any more general purposes.

\section{REFERENCES}

Aubert A, Jeulin D (2000). Estimation of the influence of second and third order moments on random sets reconstructions. Pattern Recogn 33(6):1083-103.

Barbe F, Decker L, Jeulin D, Cailletaud G (2001). Intergranular and intragranular behavior of polycristalline aggregates. Part 1: F.E. model. Int J Plasticity 17(4):513-36.
Bretheau Th, Jeulin D (1989). Caractéristiques morphologiques des constituants et comportement à la limite élastique d'un matériau biphasé $\mathrm{Fe} / \mathrm{Ag}$. Revue Phys Appl 24:861-9.

Charollais F, Bauer M, Coster M, Jeulin D, Trotabas M (1997). Modelling the structure of a nuclear ceramic obtained by solid phase sintering. Acta Stereol 16(3):315-21.

Decencière E, Jeulin D (2001). Morphological decomposition of the surface of an internal combustion engine cylinder in view of characterizing wear. Wear 249:482-8.

Decker L, Jeulin D, Tovena I (1998). 3D morphological analysis of the connectivity of a porous medium. Acta Stereol 17(1):107-12.

Decker L, Jeulin D (1999). 3D Spatial time structure simulations by reaction-diffusion models. Acta Stereol 18(2):247-54.

Decker L (1999). Modèles de Structures Aléatoires deType Réaction-Diffusion. PhD Thesis, Paris School of Mines.

Decker L, Jeulin D (2000). Simulation 3D de matériaux aléatoires polycristallins. Revue de Métallurgie CIT/Science et Génie des Matériaux, Feb. 2000: 271-5.

Delarue A (2001). Prévision du comportement électromagnétique de matériaux composites à partir de leur mode d'élaboration et de leur morphologie. $\mathrm{PhD}$ thesis, Paris School of Mines.

Delisée Ch, Jeulin D, Michaud F (2001). Caractérisation morphologique et porosité en 3D de matériaux fibreux cellulosiques. C.R. Académie des Sciences de Paris, t. 329, Série II b: 179-85.

Eyre, DJ, Milton GW (1999). A fast numerical scheme for computing the response of composites using grid refinement. Eur Phys J Appl Phys 6:41-7.

Fricout G, Cullen Mc Ewen L, Harper IS, Jeulin D, Bertram JF (2002). A quantitative method for analysing 3-D branching in embryonic kidneys; development of a technique and preliminary data. Image Anal Stereol, 21(1):37-41.

Gille W (2002). The set covariance of a dead leaves model. Adv Appl Prob (SGSA) 34:11-20.

Jeulin D, Jeulin P (1981). Synthesis of Rough Surfaces by Random Morphological Models. In: Proc. 3rd European Symposium of Stereology, Stereol Iugosl 3(1):239-46.

Jeulin D (1987). Random structure analysis and modelling by Mathematical Morphology. In: Spencer AJM, ed, Proc. CMDS5, Rotterdam: Balkema, 217-26.

Jeulin D (1989). Morphological Modeling of images by Sequential Random Functions. Signal Process 16:40331. 
Jeulin D (1991). Modèles morphologiques de structures aléatoires et de changement d'échelle. Thèse de Doctorat d'Etat, University of Caen.

Jeulin D (1993). Random models for the morphological analysis of powders. J Microsc 172(1):13-21.

Jeulin D (1994). Random structure models for composite media and fracture statistics. In: Markov $\mathrm{KZ}$, ed. Advances in Mathematical Modelling of Composite Materials. World Scientific Company (Advances in Mathematics for Applied Sciences, vol 15), 1994, 23989.

Jeulin D, Le Coënt A (1996). Morphological modeling of random composites. In: Markov KZ, ed. Proceedings of the CMDS8 Conference (Varna, 11-16 June 1995). Singapore: World Scientific Company, 199-206.

Jeulin D, Laurenge P (1996a). Probabilistic model of rough surfaces obtained by electro-erosion. In: Maragos P, Schafer RW, Butt MA, eds. Proc. ISMM'96 Conference, Atlanta, USA (11-13 May 1996). Kluwer Academic Publishers, 289-96.

Jeulin D, Laurenge P (1996b). Morphological simulation of the roughness transfer on steel sheets. Microscopy, Microanalysis, Microstructure, Vol. 7: 541-7.

Jeulin D, ed (1997). Proceedings of the Symposium on the Advances in the Theory and Applications of Random Sets (Fontainebleau, 9-11 October 1996). Singapore: World Scientific Publishing Company.

Jeulin D, Savary L (1997). Effective Complex Permittivity of Random Composites. J Phys I, sect. Cond Matt: 1123-42.

Jeulin D (1998a). Morphological modeling of surfaces. Surface Eng 14(3):199-204.

Jeulin D (1998b). Probabilistic models of structures. Invited Key-Note Lecture, Workshop PROBAMAT 21rst Century, Perm, Russia, 10-12 September 1997. In: Frantziskonis GN (ed). NATO ASI Series, vol. 46: 23357.

Jeulin D (2000a). Random texture models for materials structures. Statistics and Computing, 10: 121-31.

Jeulin D (2000b). Models of random damage. In: Miannay D, Costa P, François D, Pineau A, eds. Proc.Euromat 2000 Conference, Tours, France: 771-6.
Jeulin D (2001). Random Structure Models for Homogenization and Fracture Statistics. In: Jeulin D and Ostoja-Starzewski M, eds. Mechanics of Random and Multiscale Microstructures (CISM Lecture Notes $\mathrm{N}^{\circ}$ 430). Springer Verlag, 33-91.

Jeulin D, Monnaie P, Péronnet F (2001). Gypsum morphological analysis and modeling. Cement and Concrete Composites, Vol. 23/2-3: 299-311.

Kanit T, Forest S, Galliet I, Mounoury V, Jeulin D (2002). Determination of the Representative Volume Element for random composites: statistical and numerical approach. Submitted to International Journal of Solids and Structures.

Lantuéjoul Ch (2002). Geostatistical Simulation. Models and Algorithms. Springer Verlag.

Matheron G (1967). Eléments pour une théorie des milieux poreux. Paris: Masson.

Matheron G(1969). Théorie des Ensembles Aléatoires. Cahiers du Centre de Morphologie Mathématique, Fascicule 4, Paris School of Mines publication.

Matheron G (1975). Random sets and integral geometry. New York: J. Wiley.

Moulinec H, Suquet P (1994). A fast numerical method for computing the linear and nonlinear mechanical properties of composites. Compt Rend Acad Sci Paris, 318, Série II: 1417-23.

Norberg T (1986). Random capacities and their distributions. Prob Theo Relat Fields, 73:281-97.

Savary L, Jeulin D, Thorel A (1999). Morphological analysis of carbon-polymer composite materials from thick sections. Acta Stereologica, 18/3: 297-303.

Serra J (1982). Image analysis and mathematical morphology. London: Academic Press.

Serra J (1988). Image analysis and mathematical morphology, Vol. 2. London: Academic Press.

Stoyan D, Kendall WS, Mecke J (1987). Stochastic Geometry and its Applications. New York: J. Wiley.

Vervaat W (1988). Narrow and vague convergence of set functions. Statistics and Probability Letters, 6: 295-8. 